\title{
Ultrasonographic and cytopathological characteristics of thyroid nodules in Graves' disease
}

\section{Cuneyd Anil, Nazli Kirnap, Ozlem Turhan Iyidir, Neslihan Bascil Tutuncu \\ Baskent University, Faculty of Medicine, Department of Endocrinology and Metabolism}

\section{Objectives:}

The risk of malignancy has often been reported to be more frequent in Graves' disease (GD) than nonautoimmune thyroid nodules. However, frequency of thyroid nodules, diagnostic yield of fine needle aspiration (fna), and their potential for bearing malignancy in GD are still debatable issues (1-3). We aimed to determine the prevalence, ultrasonographic and cytological features, and the rate of malignancy outcome of thyroid nodules in a group of patients with GD.
This was a single center retrospective study. All patients with GD followed up in the outpatient clinic of Department of Endocrinology between 2008 and 2013 were included. Thyroid hormone, thyroid autoantibody, TSH receptor antibody (TRAb) levels of all cases during diagnosis were recorded. Basic ultrasonographic data, cytological results of every nodule which underwent fna, and the histopathological data were also recorded.

\section{Results:}

175 cases ( 132 female) with mean \pm SD age $45.9 \pm 15.8$ were included. $58.8 \%$ of the whole group were TRAb positive. $81.1 \%(n=142)$ of the patients were treated with antithyroid drugs. $14.3 \%(n=25)$ were finally treated with radioactive iodine with a median (min-max) dose of $12 \mathrm{mCi}$ (5$20) .40 \%(n=70)$ of the cases had totally 134 thyroid nodules. The median (min-max) value of maximum diameter of thyroid nodules was 10.0 (3-54) $\mathrm{mm} .14 .8 \%$ of the nodules owned microcalcifications. $23 \%(n=28)$ nodules underwent fna. Cytopathology revealed that 24 were benign, two were indeterminate, and the last two were non-diagnostic, both of which were also eventually benign in repeat aspirations. The indeterminate nodules went to surgery, which turned out to be benign. Eight additional patients underwent surgery for ablative purposes; only one turned out to be malignant, which was incidental.

\section{Conclusions:}

Nodular goiter is prevalent in GD. Diagnostic yield of fine needle aspiration may not be so low; malignancy rates do not seem to be high in this group of patients. 